Check for updates

Cite this: RSC Adv., 2019, 9, 31806

Received 26th August 2019

Accepted 1st October 2019

DOI: $10.1039 / \mathrm{c} 9 \mathrm{ra06728 \textrm {k }}$

rsc.li/rsc-advances

\title{
Self-healing zwitterionic sulfobetaine
nanocomposite hydrogels with good mechanical \\ Self-healing zwitterionic sulfobetaine
nanocomposite hydrogels with good mechanical properties $\dagger$
}

\author{
Yinlei Lin, (D) *ab Zheng Zeng, ${ }^{\mathrm{b}}$ Yuhao Li, ${ }^{a}$ Sheng Sun, ${ }^{a}$ Xiaoting Liu, ${ }^{a}$ Deliu He \\ and Guangji Li iD *b
}

\begin{abstract}
The development of zwitterionic hydrogels possessing both excellent self-healing and mechanical properties is of great significance. Herein, a class of zwitterionic sulfobetaine nanocomposite hydrogels was prepared by UV-initiated copolymerisation of zwitterionic sulfobetaine monomer $N, N$-dimethyl- $N$ (3-methacrylamidopropyl)- $N$-(3-sulfopropyl) ammonium betaine (DMAPMAPS) and 2-hydroxyethyl methacrylate (HEMA) in the presence of exfoliated clay platelets uniformly dispersed in an aqueous medium. The effects of the hydrogel compositions, including the DMAPMAPS/HEMA mass ratio and the amount of clay, on the self-healing behaviors and mechanical properties of the nanocomposite hydrogels were investigated. The results indicate that the fabricated zwitterionic sulfobetaine nanocomposite hydrogels can autonomously repair incisions or cracks at ambient temperature without the need for any stimulus and possess excellent mechanical properties.
\end{abstract}

\section{Introduction}

Zwitterionic betaine polymers represent a strongly developing class of polymers. ${ }^{1}$ They are ionic polymers containing both positive and negative charges in their pendant groups. ${ }^{2}$ Zwitterionic betaine polymeric materials, mainly including poly(phosphorylcholine) (PMPC), poly(sulfobetaine) (PSB) and ploy(carboxybetaine) (PCB), can exhibit some unique biological properties such as non-cytotoxicity, ${ }^{3,4}$ low endotoxin levels, ${ }^{4}$ ultra-low fouling ${ }^{5,6}$ or high resistance to non-specific protein adsorption ${ }^{7,8}$ and cell adhesion. ${ }^{9,10}$ The monomers for preparing zwitterionic betaine polymers possess various chemical structures and include, most typically, sulfobetaines, carboxybetaines and phosphorylbetaines. Among them, the sulfobetaine monomer is frequently used due to its low price, commercial availability and synthetic accessibility. ${ }^{\mathbf{1 1}}$ Therefore, zwitterionic sulfobetaine polymers have become the most widely studied zwitterionic polymer because they can be easily prepared. ${ }^{12}$ In addition to the above-mentioned biological properties, zwitterionic sulfobetaine polymers are generally thermosensitive in aqueous solutions and exhibit upper critical solution temperature (UCST) phase transitions. ${ }^{13-15}$

${ }^{a}$ School of Materials Science and Energy Engineering, Foshan University, Foshan, Guangdong 528000, China. E-mail: linyinlei@fosu.edu.cn

${ }^{b}$ School of Materials Science and Engineering, South China University of Technology, Guangzhou, Guangdong 510640, China

$\dagger$ Electronic supplementary information (ESI) available. See DOI: 10.1039/c9ra06728k
The zwitterionic betaine hydrogels, formed by virtue of a strong ability of zwitterionic moieties to bind water molecules, will have various potential applications, such as soft contact lenses, ${ }^{16,17}$ wound dressing, ${ }^{3,18,19}$ drug release ${ }^{20-22}$ and non-fouling materials. ${ }^{23,24}$ It implies that zwitterionic betaine hydrogels will hold great application potentials as biomaterials. Zwitterionic betaine hydrogels, consisting of phosphobetaine, sulfobetaine, or carboxybetaine polymers, are also of interest as a new type of hydrogels. ${ }^{25,26}$ They can be prepared through initiating a mixed solution containing a zwitterionic betaine monomer and a crosslinker. Due to their excellent biocompatibility, anti-biofouling and other unique properties, much effort has been devoted to synthesize zwitterionic betaine hydrogels. However, like most of the polymeric hydrogels, zwitterionic betaine hydrogels also exhibit poor mechanical properties, thus limiting their applications. To solve this issue, several types of zwitterionic betaine hydrogels with excellent mechanical properties have been developed by endowing them with some unique structures, such as an interpenetrating network, ${ }^{\mathbf{1 4 , 2 7}}$ a chemical-physical double network, ${ }^{28}$ or a nanocomposite structure. ${ }^{29}$

Recently, self-healing properties have been extended to zwitterionic betaine hydrogels. ${ }^{30-32}$ This opens up a new way to construct novel zwitterionic betaine hydrogels with excellent mechanical properties and unique functions. It has been recognized that natural biomaterials like skin tissue, cartilage or tendon have a long lifetime because they can heal damage autonomously and spontaneously and not because they repeatedly increase their resistance to failure. ${ }^{33}$ Thus, selfhealing hydrogels, one of the most important self-healing polymeric materials, have attracted extensive attention over 
the past decades. ${ }^{\mathbf{3 0 , 3 4}}$ Definitely, it is of great significance to design and fabricate novel zwitterionic betaine hydrogels possessing both self-healing and excellent mechanical properties.

Current advances in the hydrogel fabrication have resulted in different strategies for development of self-healing and mechanically robust hydrogels. ${ }^{30,31,35-38}$ One such approach is the preparation of nanocomposite hydrogels consisting of polymer chains that are physically cross-linked with nanoparticles such as inorganic clay LAPONITE® XLG. ${ }^{29,30,37}$ The self-healing capability of nanocomposite hydrogels can be attributed to the unique polymer/clay network structure. The interaction between the polymer chains and the adjacent clay particles is considered to be a non-covalent bonding or hydrogen bonding. By adjoining the two such surfaces, grafted chains at the two surfaces diffuse into each other and interact with neighboring clay platelets by hydrogen bonding. Consequently, a number of new crosslinks form across the interface, which leads the cut surfaces to rejoin. Thus, the macroscopic self-healing of nanocomposite hydrogels arises from the reconstruction of the network at the interface due to mutual diffusion of long grafted chains and their subsequent interactions with clay. ${ }^{38}$

Here we present a facile method to prepare zwitterionic sulfobetaine nanocomposite hydrogels with self-healing, highly stretchable and mechanically tough properties. As schematically illustrated in Fig. 1, the zwitterionic sulfobetaine nanocomposite hydrogels were prepared by UV-initiated copolymerisation of zwitterionic sulfobetaine monomer $\mathrm{N}, \mathrm{N}$ dimethyl- $N$-(3-methacrylamidopropyl)- $N$-(3-sulfopropyl) ammonium betaine (DMAPMAPS) and 2-hydroxyethyl methacrylate (HEMA) in the presence of uniformly dispersed clay nanoparticles (LAPONITE® XLG) in water. The morphology, chemical composition and properties of the zwitterionic sulfobetaine nanocomposite hydrogels with different chain compositions were measured and analyzed.

\section{Experimental}

\section{Materials}

$N$-(3-dimethylaminopropyl)methacrylamide (DMAPMA, 99\%), 2hydroxyethyl methacrylate (HEMA, 96\%) were purchased from Aladdin and used as received. 1,3-Propane sultone (1,3-PS, 99.9\%) was purchased from Shandong Yinghuan Chemical Co., Ltd. 2Hydroxy-4-(2-hydroxyethoxy)-2-methylpropiophe-none (Irgacure

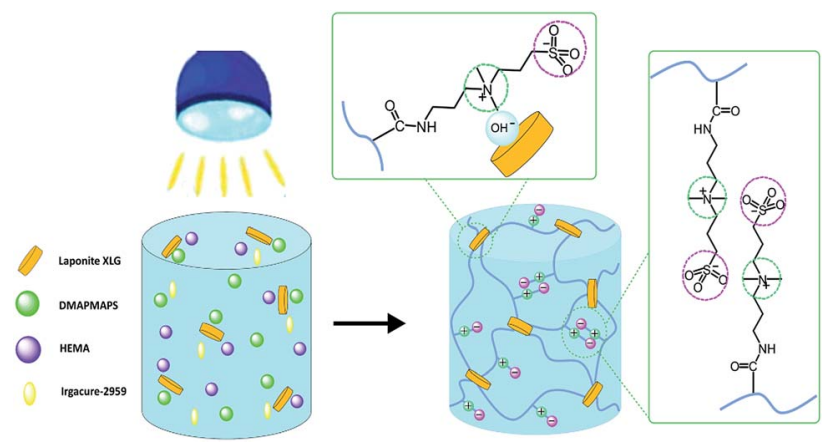

Fig. 1 Schematic illustration to the synthesis of zwitterionic sulfobetaine nanocomposite hydrogels.
2959, 98\%) was purchased from Aldrich. Inorganic clay “LAPONITE® XLG" nanoparticles $\left[\mathrm{Mg}_{5.34} \mathrm{Li}_{0.66} \mathrm{Si}_{8} \mathrm{O}_{20}(\mathrm{OH})_{4}\right] \mathrm{Na}_{0.66}$ (30 and $1 \mathrm{~nm}$ in diameter and thickness, respectively) were purchased from Rockwood Ltd, UK. All of the other reagents and solvents were of analytical grade and used without further purification, unless indicated otherwise.

\section{Synthesis of $N, N$-dimethyl- $N$-(3-methacrylamidopropyl)- $N$-(3- sulfopropyl) ammonium betaine (DMAPMAPS) monomer}

The $N, N$-dimethyl- $N$-(3-methacrylamidopropyl)- $N$-(3-sulfopropyl) ammonium betaine (DMAPMAPS) monomer was synthesized via the reaction of DMAPMA and 1,3-PS according to our previous work. ${ }^{39}$ A mixture of 1,3-PS (6.41 g, $\left.0.05 \mathrm{~mol}\right)$ and acetone (15 g) was added dropwise into a three-necked flask containing a mixture of DMAPMA ( $8.51 \mathrm{~g}, 0.05 \mathrm{~mol})$ and acetone $(15 \mathrm{~g})$ under stirring over a total time span of $0.5 \mathrm{~h}$. Then the reaction mixture was stirred at room temperature for $24 \mathrm{~h}$. The final white powdery product was filtered, washed with acetone, and dried under reduced pressure for $24 \mathrm{~h}$ at $40{ }^{\circ} \mathrm{C}$. (Yield: 93\%).

FTIR (KBr pellet, $\left.\mathrm{cm}^{-1}\right): 3320(\mathrm{~N}-\mathrm{H}$, stretch); $3030(=\mathrm{C}-\mathrm{H}$, stretch); 2976, 2928, $2877\left(-\mathrm{CH}_{3},-\mathrm{CH}_{2}-\right.$, stretch); $1653(\mathrm{C}=\mathrm{O}$, stretch); 1611 ( $\mathrm{C}=\mathrm{C}$, stretch); 1543 (N-H, in-plane bend); 1484 $\left(\mathrm{N}^{+}-\mathrm{C}\right.$, stretch); 1193 ( $\mathrm{S}=\mathrm{O}$, deformation); 1034 ( $\mathrm{S}=\mathrm{O}$, deformation) (ESI, Fig. $\mathrm{S} 1 \dagger)$.

${ }^{1} \mathrm{H}-\mathrm{NMR}\left(400 \mathrm{MHz}, \mathrm{D}_{2} \mathrm{O}, \delta\right): 5.66 \mathrm{ppm}\left(1 \mathrm{H}, \mathrm{CH}_{2}=\mathrm{CH}-\mathrm{CO}-\right)$; $5.42 \mathrm{ppm}\left(1 \mathrm{H}, \mathrm{CH}_{2}=\mathrm{CH}-\mathrm{CO}-\right) ; 3.40-3.44 \mathrm{ppm}\left(2 \mathrm{H},-\mathrm{N}\left(\mathrm{CH}_{3}\right)_{2}-\right.$ $\left.\mathrm{CH}_{2}-\mathrm{CH}_{2}-\mathrm{CH}_{2}-\mathrm{SO}_{3}\right) ; 3.29-3.33$ ppm (4H, $-\mathrm{NH}-\mathrm{CH}_{2}-\mathrm{CH}_{2}-\mathrm{CH}_{2}-$ $\left.\mathrm{N}\left(\mathrm{CH}_{3}\right)_{2}-\right)$; 3.05 ppm $\left(6 \mathrm{H},-\mathrm{N}\left(\mathrm{CH}_{3}\right)_{2}-\right) ; 2.90-2.94 \mathrm{ppm}(2 \mathrm{H}$, $\left.-\mathrm{CH}_{2}-\mathrm{SO}_{3}\right) ; 2.11-2.19 \mathrm{ppm}\left(2 \mathrm{H},-\mathrm{CH}_{2}-\mathrm{CH}_{2}-\mathrm{SO}_{3}\right) ; 1.96-2.03 \mathrm{ppm}$ $\left(2 \mathrm{H},-\mathrm{NH}-\mathrm{CH}_{2}-\mathrm{CH}_{2}^{-}\right)$(ESI, Fig. $\left.\mathrm{S} 2 \dagger\right)$.

\section{Synthesis of zwitterionic sulfobetaine nanocomposite hydrogels}

Zwitterionic sulfobetaine nanocomposite hydrogels were synthesised by copolymerisation of DMAPMAPS and HEMA comonomer in the presence of exfoliated clay platelets in aqueous media. First, the "LAPONITE® XLG" nanoparticles were dispersed in water at concentrations of 3,5 and $7 \mathrm{wt} \%$. Then the HEMA and DMAPMAPS comonomer were added to the dispersed "LAPONITE® XLG" nanoparticles in the water at a concentration of $20 \mathrm{wt} \%$ (the weight ratio of DMAPMAPS/ HEMA was 20/80, 40/60 and 50/50, respectively.). Irgacure 2959 ( $2.0 \mathrm{wt} \%$, relative to the total monomer mass) was then added to the solution with stirring in the dark until complete dissolution. Subsequently, the obtained mixture solution was transferred into a cylindrical mould and irradiated with $365 \mathrm{~nm}$ UV light in a UV oven at ambient temperature for $1 \mathrm{~h}$ to initiate and perform the copolymerization of DMAPMAPS and HEMA comonomer. The resulting product was removed from the mould. All the prepared hydrogels are displayed in Fig. S3 in ESI. $\dagger$

For comparison, pure poly(HEMA) and poly(DMAPMAPS) nanocomposite hydrogels were also fabricated by the homopolymerization according to the above-described protocol. The formulations of nanocomposite hydrogels are listed in Table 1. 


\section{Characterization}

${ }^{1} \mathrm{H}$ NMR spectra were measured at ambient temperature on a Bruker Avance III 400 spectrometer (400 MHz). The sample was dissolved in deuterated water $\left(\mathrm{D}_{2} \mathrm{O}\right)$. Fourier-transform infrared (FTIR) spectra of the dry hydrogel samples were recorded on a Bruker VERTEX70 IR spectrometer. The spectra were collected at 32 scans with a spectral resolution of $4 \mathrm{~cm}^{-1}$ by the $\mathrm{KBr}$ disk method. The network structures of hydrogels were characterized by scanning electron microscopy (SEM). Water-swollen hydrogel was flash-frozen in liquid nitrogen for $5 \mathrm{~min}$ and immediately lyophilized for $6 \mathrm{~h}$, and then was subjected to Au-sputter coating and viewed with a field emission scanning electron microscope (SEM, FEI Quanta 200) at an accelerated electron energy of $3 \mathrm{kV}$. The self-healing process and deformation behavior of the prepared C-DHNC5 hydrogel was recorded using the images taken with an ordinary camera and the optical microscopy images taken with a Keyence VHX-2000 optical microscope.

\section{Determination of equilibrium water content (EWC)}

The equilibrium water content (EWC) of nanocomposite hydrogels was measured at ambient temperature using a gravimetric method. The sample was immersed in deionized water and weighed upon removing water on the surface with filter paper at prescribed time intervals until the swelling reached equilibrium. Then the sample was dried to constant weigh in a vacuum oven at $50{ }^{\circ} \mathrm{C}$. The EWC of hydrogels was defined as:

$$
\mathrm{EWC}=\frac{m_{\mathrm{eq}}-m_{\mathrm{d}}}{m_{\mathrm{eq}}} \times 100 \%
$$

where $m_{\mathrm{eq}}$ and $m_{\mathrm{d}}$ denote the equilibrium wet weight and dry weight of hydrogels, respectively. At least three specimens were taken for each measurement to calculate the mean of EWC.

\section{Measurement of mechanical properties}

Rod-shaped hydrogel samples $(5.5 \mathrm{~mm}$ in diameter and $60.0 \mathrm{~mm}$ in length) were used for the measurement of tensile mechanical properties according to our previous work. ${ }^{40}$ The tension tests were carried out using a universal tensile machine (PST, Am Cells Electrion Weighing Company, USA) at ambient temperature. The cross-head speeds of the tensile measurements were $50 \mathrm{~mm} \mathrm{~min}{ }^{-1}$. At least three specimens were tested for each sample.

Table 1 Formulations of the LAPONITE $®$ XLG/HEMA/DMAPMAPS nanocomposite hydrogels as a function of the weight ratio of monomers and the concentration of LAPONITE $\circledast$ nanoparticles

\begin{tabular}{llll}
\hline & \multicolumn{3}{l}{$\begin{array}{l}\text { Concentration of LAPONITE® XLG } \\
\text { nanoparticles, wt\% }\end{array}$} \\
\cline { 2 - 4 } $\begin{array}{l}\text { The weight ratio } \\
\text { of DMAPMAPS/HEMA }\end{array}$ & 3 & 5 & 7 \\
\hline $0 / 100$ & A-HNC3 & A-HNC5 & A-HNC7 \\
$20 / 80$ & B-DHNC3 & B-DHNC5 & B-DHNC7 \\
$40 / 60$ & C-DHNC3 & C-DHNC5 & C-DHNC7 \\
$50 / 50$ & D-DHNC3 & C-DHNC5 & C-DHNC7 \\
$100 / 0$ & E-DNC3 & E-DNC5 & E-DNC7
\end{tabular}

\section{Quantification of the self-healing efficiency}

We utilized tensile tests to quantify the efficiency of the selfhealing effect. The cylindrical zwitterionic sulfobetaine nanocomposite hydrogel samples were cut into completely separate pieces, and then self-healing of damaged nanocomposite hydrogels was carried out by keeping the cut surfaces in contact under ambient conditions without additional treatment. The tensile test was performed on the samples after the self-healing for $10 \mathrm{~h}$. The tensile strength of the self-healed samples was recorded. The healing efficiency $(f)$ is defined as $f=\sigma / \sigma^{\prime} \times$ $100 \%$, where $\sigma^{\prime}$ and $\sigma$ are the tensile strengths of the healed hydrogel and the original hydrogel (uncut hydrogel), respectively.

\section{Results and discussion}

\section{Structural characterization of the hydrogels}

Fig. 2 displays the FTIR spectra of nanocomposite hydrogels. As shown in Fig. 2(b)-(g), the characteristic peaks at $1191 \mathrm{~cm}^{-1}$ and $1041 \mathrm{~cm}^{-1}$ was assigned to the $S=O$ asymmetric stretching vibration and symmetric stretching vibration, respectively. The FTIR spectrum of A-HNC hydrogel (Fig. 2(a)) exhibited several characteristic absorption bands at $1728 \mathrm{~cm}^{-1}(\mathrm{C}=\mathrm{O}$ stretching vibration), $1163 \mathrm{~cm}^{-1}$ (C-O-C antisymmetric vibration), and $1074 \mathrm{~cm}^{-1}$ (C-O-C stretching vibration). The increase in the amount of DMAPMAPS comonomer was in good agreement with the increase in the intensity of amide bands at $1650 \mathrm{~cm}^{-1}$ and $1535 \mathrm{~cm}^{-1}$, and the sulfonate group at $1191 \mathrm{~cm}^{-1}$ and $1041 \mathrm{~cm}^{-1}$ arising from the betaines added. Fig. 2(b)-(d) displays the FTIR spectra of B-DHNC hydrogel samples. A comparison of the B-DHNC hydrogels with those with different clay contents showed that there were almost no differences among the peaks for the B-DHNC3, B-DHNC5 and B-DHNC7 hydrogels.

\section{Swelling characterization of hydrogels}

The EWC of a hydrogel is one of the most critical factors influencing its mechanical properties and the other properties.

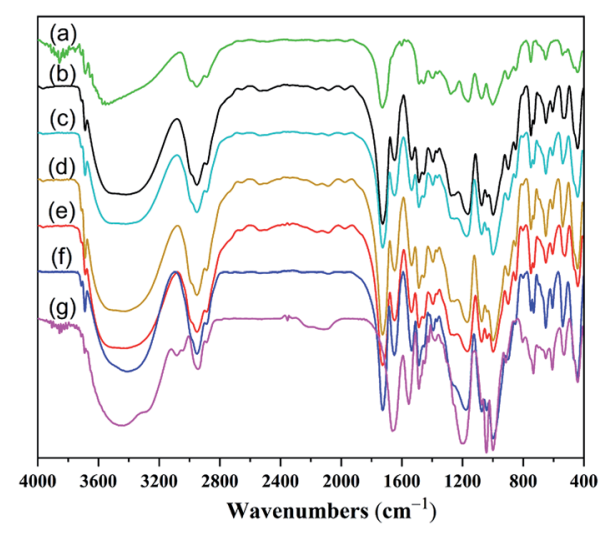

Fig. 2 FTIR spectra of nanocomposite hydrogels. A-HNC (a), BDHNC3 (b), B-DHNC5 (c), B-DHNC7 (d), C-DHNC (e), D-DHNC (f), and E-DNC (g). 
The EWC values of A-HNC, and (B-D)-DHNC hydrogel samples were measured to investigate the effect of the weight ratio of comonomers and the concentration of clay on the EWC of hydrogels. The results are shown in Fig. 3. It can be seen from Fig. 3 that EWC decreases with increasing the clay content in the hydrogels. This behavior can be explained by the clay platelets acting as effective cross-linking agents. The increase of cross-linking points results in a denser network structure and shorter interlinked polymer chains, restricting higher water uptake. It can also be seen from Fig. 3 that EWC increases with increasing the zwitterionic DMAPMAPS monomer. While AHNC hydrogel samples can only bind water via hydrogen bonding with its hydroxyl groups, (B-D)-DHNC hydrogel samples can bind water more strongly via electrostatic interactions and hydrogen bonding.

\section{Morphology of hydrogels}

To understand the swelling behaviors of zwitterionic sulfobetaine nanocomposite hydrogels, the microstructures of corresponding freeze-dried hydrogels were visualized by scanning electron microscopy (SEM). Fig. 4 shows SEM images of a crosssection of nanocomposite hydrogel samples. The zwitterionic sulfobetaine nanocomposite hydrogels exhibit large unconnected pores with a pore size of 50-200 $\mu \mathrm{m}$. The average pore sizes of the B-DHNC5 hydrogel with a low DMAPMAPS concentration are about $100 \mu \mathrm{m}$, while the pore wall is in several micrometers. The average pore sizes of D-DHNC5 hydrogel with a high DMAPMAPS concentration are about $200 \mu \mathrm{m}$, as a result of the increase in water volume in the hydrogel. As seen from the images (a), (b) and (c), the surface of pore walls of B-DHNC5 and C-DHNC5 hydrogel is smoother than that of D-DHNC5 hydrogel. By comparing the images (a) and (b), it can be seen that the morphologies of hydrogel are almost the same in the cross and vertical sections.

\section{Self-healing and stretching behaviors of hydrogels}

Fig. 5 illustrates the self-healing phenomenon of zwitterionic sulfobetaine nanocomposite hydrogel C-DHNC5. Two original rod-shaped hydrogel samples colored with and without red dyestuff were cut into two parts, respectively, as shown in

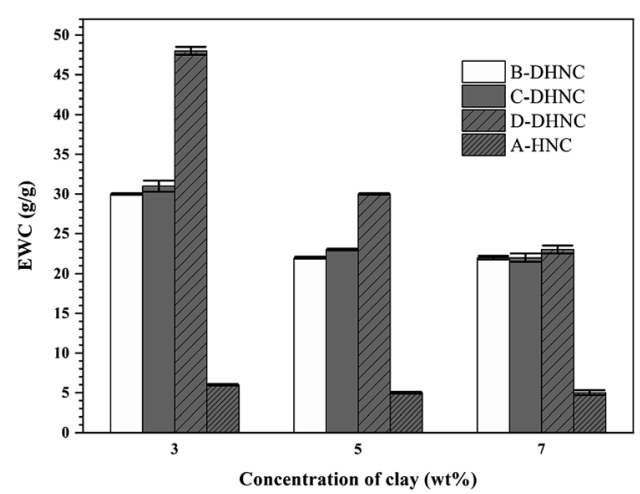

Fig. 3 EWC dependence of hydrogels on the weight ratio of comonomers and the concentration of clay.

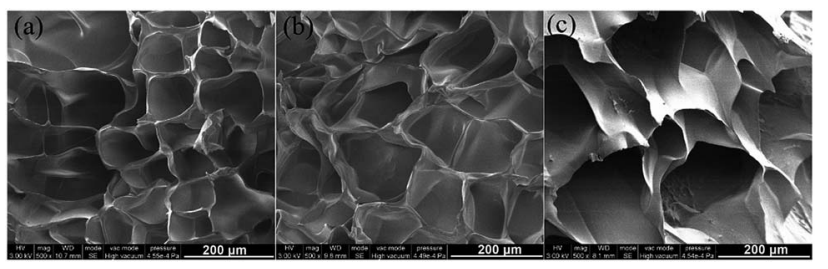

Fig. 4 The SEM micrographs of zwitterionic sulfobetaine nanocomposite hydrogels after frozen-dried, $500 \times$ magnification (the length bar is $200 \mu \mathrm{m}$ ): B-DHNC5 (a), C-DHNC5 (b) and D-DHNC5 (c).

Fig. 5(a) and (b). Then, these four 'hydrogel parts' were alternately arranged in the manner as illustrated in Fig. 5(c) and the freshly fractured surfaces of the adjacent parts with different colors were brought into contact, thus merging into a single rod-shaped hydrogel sample. After standing for $10 \mathrm{~h}$ at ambient temperature, this sample healed perfectly; its cut marks at the interfaces between two adjacent hydrogel parts disappeared almost completely, and it could be stretched by hand to a great extent without breaking, as shown in Fig. 5(d). Therefore, notable self-healing and highly stretchable ability were demonstrated.

In addition to the rod-shaped zwitterionic sulfobetaine nanocomposite hydrogel, the zwitterionic sulfobetaine nanocomposite hydrogel film can also exhibit self-healing behavior. Fig. 6 illustrates the optical microscopy images reflecting the self-healing process of the incision generated by deliberately cutting the C-DHNC5 film. The observations indicate that the incision on the C-DHNC5 hydrogel film can autonomously selfheal within $10 \mathrm{~h}$ at ambient temperature without the need for any stimulus or healing agent.

\section{Mechanical properties of hydrogels}

To further quantitatively evaluate the mechanical properties of the zwitterionic sulfobetaine nanocomposite hydrogels, tension

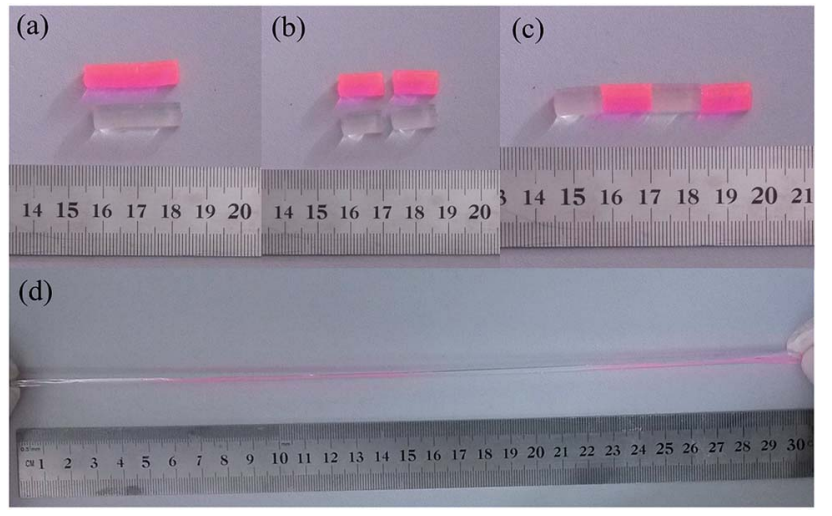

Fig. 5 Images demonstrating the self-healing and stretching behaviours of C-DHNC5 hydrogel. (a) Two original rod-shaped hydrogel samples with and without red dyestuff; (b) the four hydrogel samples obtained by cutting the original hydrogel samples; (c) self-healed hydrogel sample upon bringing the four separate halves in contact for $10 \mathrm{~h}$ in air at ambient temperature; (d) the stretching behaviour of the self-healed hydrogel sample. 


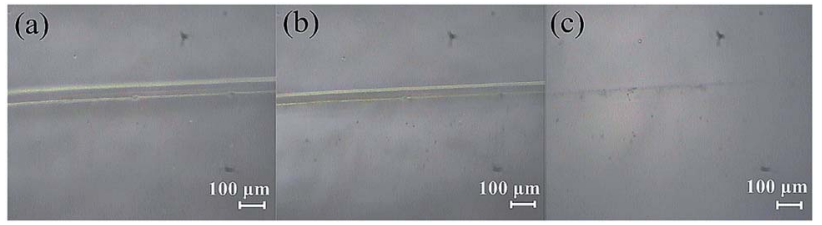

Fig. 6 Optical microscopy images that record changes of the incision (width: $\sim 80 \mu \mathrm{m}$ ) on the C-DHNC5 hydrogel film over time at ambient temperature. (a) $0 \mathrm{~h}$; (b) $5 \mathrm{~h}$; (c) $10 \mathrm{~h}$.

tests were performed on the original (uncut) samples and the self-healed samples upon bringing the two separate parts in contact for $10 \mathrm{~h}$. The tensile stress-strain curves of the original and self-healed hydrogel samples, and the tensile strength recovery of the self-healed hydrogel are shown in Fig. 7(a)-(c) and Fig. 7(d), respectively. The corresponding values are summarized in Table S1. $\dagger$ It can be seen from Fig. 7(a)-(c) that the original hydrogels and the corresponding self-healed nanocomposite hydrogels exhibit similar stress-strain behaviors and great stretchability; and their tensile strength increases with increasing the clay content and HEMA comonomer content in nanocomposite hydrogels. To be exact, the tensile strength and elongation at break of the original zwitterionic sulfobetaine nanocomposite hydrogels containing 3.0 to $7.0 \mathrm{wt} \%$ clay range from 67.0 to $168.0 \mathrm{kPa}$ and from 600 to $2300 \%$, respectively. The corresponding nanocomposite hydrogels self-healed for $10 \mathrm{~h}$ possess a slightly lower tensile stress at the same strain level than the original hydrogels, but their tensile strength still lies in the range from 57 to $146.0 \mathrm{kPa}$, corresponding to $85-95 \%$ of the tensile strength of the original hydrogels. This is a significant result for certain hydrogel applications.

The robustness and toughness of the zwitterionic sulfobetaine nanocomposite hydrogels were clearly embodied using
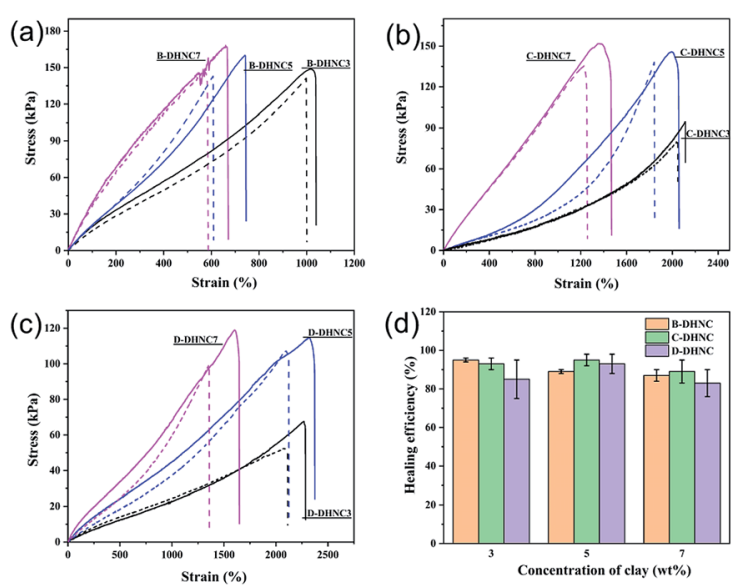

Fig. 7 (a-c) Tensile stress-strain curves of the original zwitterionic sulfobetaine nanocomposite hydrogel samples (solid line) and the corresponding self-healed zwitterionic sulfobetaine nanocomposite hydrogel samples at $10 \mathrm{~h}$ healing time (dotted line). (d) The healing efficiency of self-healed the zwitterionic sulfobetaine nanocomposite hydrogel.

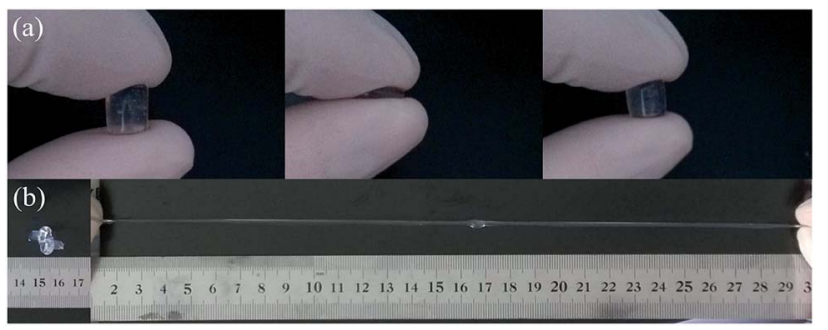

Fig. 8 Images demonstrating the robustness of the prepared zwitterionic sulfobetaine nanocomposite hydrogels. (a) Deformation and recovery of $\mathrm{C}$-DHNC5 hydrogel under stress; (b) the original knot tied with a C-DHNC5 hydrogel strip (left) and its stretched state (right).

the images recording a specially designed intuitive demonstration, as shown in Fig. 8 and S4. $\dagger$ In Fig. 8(a), the hydrogel CDHNC5 exhibited large deformation under stress and strong ability to recover its original shape upon removal of stress similar to an elastomer. Fig. 8(b) illustrates the highly stretchable property of the knot tied with a C-DHNC5 hydrogel strip and its excellent ability to withstand knotting. Thus, the robustness of the prepared zwitterionic sulfobetaine nanocomposite hydrogels is fully confirmed.

The self-healing mechanism of the prepared zwitterionic sulfobetaine hydrogel is depicted in Fig. 1. The self-healing properties of the hydrogels can be attributed to the ionic interactions between the zwitterion and clay platelets. The zwitterionic sulfobetaine polymer chains can interact with the clay platelets by ionic interactions and form self-association, which can form the physically cross-linked networks. ${ }^{37}$ The reversible interactions in hydrogels can endow the hydrogels with self-healing properties. Moreover, the presence of the zwitterionic electrostatic interactions between intrinsic sulfobetaine groups on zwitterionic sulfobetaine polymer chains also contributes to the healing potential of the zwitterionic sulfobetaine hydrogel. ${ }^{31}$

\section{Conclusions}

A class of zwitterionic sulfobetaine nanocomposite hydrogels was successfully fabricated by the copolymerization of zwitterionic sulfobetaine monomer (DMAPMAPS) with the HEMA monomer. The obtained zwitterionic sulfobetaine nanocomposite hydrogels can autonomously repair occurring incisions or cracks within $10 \mathrm{~h}$ at ambient temperature without the need for any stimulus, thereby exhibiting outstanding selfhealing ability. They also exhibit excellent elasticity, robustness and toughness under tensile stress. The tensile strength and elongation at break of the original zwitterionic sulfobetaine nanocomposite hydrogels containing 3.0 to $7.0 \mathrm{wt} \%$ clay are found in the range from 67.0 to $168.0 \mathrm{kPa}$ and from 600 to $2300 \%$, respectively. These properties of the prepared hydrogels are essential for practical applications.

\section{Conflicts of interest}

There are no conflicts to declare. 


\section{Acknowledgements}

This work was supported by the National Natural Science Foundation of China (51803028) and the scientific research start-up fund for high-level talents of Foshan University, China (gg07077), the Science and Technology Planning Project of Guangdong Province, China (2016A010103005) and the Key Project of Department of Education of Guangdong Province (2016GCZX008).

\section{Notes and references}

1 L. D. Blackman, P. A. Gunatillake, P. Cass and K. E. S. Locock, Chem. Soc. Rev., 2019, 48, 757-770.

2 A. B. Lowe and C. L. McCormick, Chem. Rev., 2002, 102, 4117-4189.

3 K. Huang, Y. Fang, P. Hsieh, C. Li, N. Dai and C. Huang, J. Mater. Chem. B, 2016, 4, 11515-11522.

4 Z. Zhang, T. Chao, L. Liu, G. Cheng, B. Ratner and S. Jiang, J. Biomater. Sci., Polym. Ed., 2009, 20, 1845-1859.

5 L. Zhang, Z. Cao, T. Bai, L. Carr, J. Ella-Menye, C. Irvin, B. D. Ratner and S. Jiang, Nat. Biotechnol., 2013, 31, 553-556. 6 S. Jiang and Z. Cao, Adv. Mater., 2010, 22, 920-932.

7 L. Ye, Y. Zhang, Q. Wang, X. Zhou, B. Yang, F. Ji, D. Dong, L. Gao, Y. Cui and F. Yao, ACS Appl. Mater. Interfaces, 2016, 24, 15710-15723.

8 L. Carr, H. Xue and S. Jiang, Biomaterials, 2011, 32, 961-968. 9 L. Mi and S. Jiang, Biomaterials, 2012, 33, 8928-8933.

10 H. W. Chien, X. Xu, J. R. Ella-Menye, W. B. Tsai and S. Jiang, Langmuir, 2012, 28, 17778-17784.

11 P. Kasák, Z. Kroneková, I. Krupa and I. Lacík, Polymer, 2011, 52, 3011-3020.

12 Y. Chang, W. Yandi, W. Chen, Y. Shih, C. Yang, Y. Chang, Q. Ling and A. Higuchi, Biomacromolecules, 2010, 11, 11011110.

13 J. Seuring and S. Agarwal, Macromol. Rapid Commun., 2012, 33, 1898-1920.

14 L. Chen, J. P. Gong and Y. Osada, Macromol. Rapid Commun., 2002, 23, 171-174.

15 L. P. Kreuzer, T. Widmann, N. Hohn, K. Wang, L. Bießmann, L. Peis, J. Moulin, V. Hildebrand, A. Laschewsky, C. M. Papadakis and P. Müller-Buschbaum, Macromolecules, 2019, 9, 3486-3498.

16 J. Wu, C. He, H. He, C. Cheng, J. Zhu, Z. Xiao, H. Zhang, X. Li, J. Zheng and J. Xiao, J. Mater. Chem. B, 2017, 5, 4595-4606.

17 A. Xiao, C. Dhand, C. M. Leung, R. W. Beuerman, S. Ramakrishna and R. Lakshminarayanan, J. Mater. Chem. $B, 2018,6,2171-2186$.
18 K. Huang, Y. Fang, P. Hsieh, C. Li, N. Dai and C. Huang, Biomater. Sci., 2017, 5, 1072-1081.

19 Y. Zhu, J. Zhang, J. Yang, C. Pan, T. Xu and L. Zhang, J. Mater. Chem. B, 2016, 4, 5105-5111.

20 G. Ma, W. Lin, Z. Yuan, J. Wu, H. Qian, L. Xu and S. Chen, J. Mater. Chem. B, 2017, 5, 935-943.

21 X. Chen, X. Qiu, M. Hou, X. Wu, Y. Dong, Y. Ma, L. Yang and Y. Wei, Langmuir, 2019, 5, 1475-1482.

22 J. Park, E. S. Jeong, S. Kim, Y. Kim, H. Jeong and J. W. Kim, Colloid Polym. Sci., 2015, 4, 1121-1127.

23 B. Yang, C. Wang, Y. Zhang, L. Ye, Y. Qian, Y. Shu, J. Wang, J. Li and F. Yao, Polym. Chem., 2015, 6, 3431-3442.

24 H. He, X. Xuan, C. Zhang, Y. Song, S. Chen, X. Gong, B. Ren, J. Zheng and J. Wu, Langmuir, 2019, 5, 1828-1836.

25 Q. Shao and S. Jiang, Adv. Mater., 2015, 27, 15-26.

26 A. Ali and S. Ahmed, J. Agric. Food Chem., 2018, 66, 69406967.

27 Y. Iwasaki, K. Shimakata, N. Morimoto and K. Kurita, J. Polym. Sci., Part A: Polym. Chem., 2002, 41, 68-75.

28 Z. Zhang, T. Chao and S. Jiang, J. Phys. Chem. B, 2008, 112, 5327-5332.

29 J. Ning, G. Li and K. Haraguchi, Macromolecules, 2013, 46, 5317-5328.

30 N. Y. Kostina, S. Sharifi, A. de los Santos Pereira, J. Michálek, D. W. Grijpma and C. Rodriguez-Emmenegger, J. Mater. Chem. B, 2013, 1, 5644-5650.

31 T. Bai, S. Liu, F. Sun, A. Sinclair, L. Zhang, Q. Shao and S. Jiang, Biomaterials, 2014, 35, 3926-3933.

32 A. Sinclair, M. B. O'Kelly, T. Bai, H. Hung, P. Jain and S. Jiang, Adv. Mater., 2018, 30, 1803087.

33 S. Billiet, X. K. D. Hillewaere, R. F. A. Teixeira and F. E. Du Prez, Macromol. Rapid Commun., 2013, 34, 290-309.

34 Q. Wang, J. L. Mynar, M. Yoshida, E. Lee, M. Lee, K. Okuro, K. Kinbara and T. Aida, Nature, 2010, 463, 339-343.

35 W. Diao, L. Wu, X. Ma, Z. Zhuang, S. Li, X. Bu and Y. Fang, J. Appl. Polym. Sci., 2019, 136, 47783.

36 Y. Shi, Y. Zhang, L. Jia, Q. Zhang and X. Xu, ACS Appl. Mater. Interfaces, 2018, 42, 36028-36036.

37 L. Wang, G. Gao, Y. Zhou, T. Xu, J. Chen, R. Wang, R. Zhang and J. Fu, ACS Appl. Mater. Interfaces, 2019, 3, 3506-3515.

38 K. Haraguchi, J. Ning and G. Li, Macromol. Symp., 2015, 358, 182-193.

39 L. Wang, G. Li, Y. Lin, Z. Zhang, Z. Chen and S. Wu, Polym. Chem., 2016, 7, 4964-4974.

40 Y. Lin, D. He, Z. Chen, L. Wang and G. Li, RSC Adv., 2016, 6, 12479-12485. 